This is an author produced version of a paper published in Computer Physics Communications. This paper has been peer-reviewed but does not include the final publisher proof-corrections or journal pagination.

Citation for the published paper:

Naze, Cedric; Gaidamauskas, Erikas; Gaigalas, Gediminas; Godefroid, Michel; Jönsson, Per. (2013). RIS3 : a program for relativistic isotope shift calculations. Computer Physics Communications, vol. 184, issue 9, p. null

URL: https://doi.org/10.1016/j.cpc.2013.02.015

Publisher: Elsevier

This document has been downloaded from MUEP (https://muep.mah.se) / DIVA (https://mau.diva-portal.org). 


\title{
RIS3: a program for relativistic isotope shift calculations
}

\author{
C. Nazéa ${ }^{a}$, E. Gaidamauskas ${ }^{\mathrm{b}}$, G. Gaigalas ${ }^{\mathrm{b}}$, M. Godefroid ${ }^{\mathrm{a}}$, P. Jönsson ${ }^{\mathrm{c}}$ \\ ${ }^{a}$ Chimie Quantique et Photophysique, CP160/09, Université Libre de Bruxelles, \\ Av. F.D. Roosevelt 50, B-1050 Brussels, Belgium \\ ${ }^{b}$ Vilnius University, Institute of Theoretical Physics and Astronomy, \\ A. Goštauto 12, LT-01108 Vilnius, Lithuania \\ ${ }^{c}$ School of Technology, Malmö University, S-20506 Malmö, Sweden
}

\begin{abstract}
An atomic spectral line is characteristic of the element producing the spectrum. The line also depends on the isotope. The program Ris3 (Relativistic Isotope Shift) calculates the electron density at the origin and the normal and specific mass shift parameters. Combining these electronic quantities with available nuclear data, isotope-dependent energy level shifts are determined.
\end{abstract}

Keywords: Isotope shift, field shift, mass shift, normal mass shift, specific mass shift, relativistic nuclear recoil, nuclear radius

\section{NEW VERSION PROGRAM SUMMARY}

Manuscript Title: RIS3: a program for relativistic isotope shift calculations Authors:??

Program Title: RIS3

Journal Reference:??

Catalogue identifier:??

Licensing provisions:??

Programming language: Fortran 77

Computer: HP ProLiant BL465c G7 CTO

Operating system: Centos 5.5, which is a Linux distribution compatible with Red Hat Enterprise Advanced Server.

$R A M:$ ?? bytes

Supplementary material: ??none?

Keywords: Isotope shift, field shift, mass shift, normal mass shift, specific mass

Email addresses: mrgodef@ulb.ac.be (M. Godefroid), per.jonsson@mah.se (P. Jönsson) 
shift, relativistic nuclear recoil, nuclear radius

Classification: ??

External routines/libraries: ??

Subprograms used: GRASP2K version 1_1

Catalogue identifier of previous version: ADEK

Journal reference of previous version: Comput. Phys. Commun. 100 (1997) 81-92

Does the new version supersede the previous version?: Yes

Nature of problem:

Prediction of level and transition isotope shifts in atoms using four-component relativistic wave functions.

Solution method:

The nuclear motion and volume effects are treated in first order perturbation theory. Taking the zero-order wave function in terms of a configuration state expansion $\left|\Psi_{P J M_{J}}\right\rangle=\sum_{\mu} c_{\mu}\left|\Phi\left(\gamma_{\mu} P J M_{J}\right)\right\rangle$, where $P, J$ and $M_{J}$ are, respectively, the parity and angular quantum numbers, the electron density at the nucleus and the normal and specific mass shift parameters may generally be expressed as $\sum_{\mu, \nu} c_{\mu} c_{\nu}\left\langle\gamma_{\mu} P J M_{J}|\vartheta| \gamma_{\nu} P J M_{J}\right\rangle$ where $\vartheta$ is the relevant operator. The matrix elements, in turn, can be expressed as sums over radial integrals multiplied by angular coefficients. All the angular coefficients are calculated using routines from GRASP2K version 1_1 package [1].

Reasons for the new version:

This new version takes the nuclear recoil corrections into account within the $(\alpha Z)^{4} m^{2} / M$ approximation [2] and also allows a storage of the angular coefficients for a series of calculations within a given isoelectronic sequence.

Furthermore, the program JJ2LSJ, a module of GRASP2K version 1_1 toolkit that allows a transformation of ASFs from a $j j$-coupled CSF basis into an $L S J$-coupled CSF basis, has been especially adapted to present RIS3 results using $L S J$ labels of the states. This additional tool is called RIS3_LSJ.

Summary of revisions:

This version is compatible with the new angular approach of GRASP2K version 1_1 package [1] and can store necessary angular coefficients. According to the formalism of the relativistic nuclear recoil, the "uncorrected" expression of the normal mass shift has been fundamentally modified compared with its expression in [3].

Restrictions:

The complexity of the cases that can be handled is entirely determined by GRASP $2 \mathrm{~K}$ package [1] used for the generation of the electronic wave functions.

Unusual features:

Angular data stored on disc and can be reused. $L S J$ labels are used for the states. Additional comments:

?? 


\section{Running time:}

As an example, we evaluated the isotope shifts parameters and the electron density at the origin using the wave functions of Be-like system. We used MCDHF wave function built on a complete active space (CAS) with $n=8$ (296 626 CSFs - 62 orbitals) that contains 3 non-interacting blocks of given parity and $J$ values involving 6 different eigenvalues in total. Calculations take around 10 hours on one AMD Opteron $6100 @ 2.3 \mathrm{GHz}$ CPU with 8 cores (64GB DDR3 RAM 1.333GHz). If angular files are available the time is reduced to 20 minutes. The storage of the angular data takes $139 \mathrm{Mb}$ and $7.2 \mathrm{~Gb}$ for the one-body and the two-body elements, respectively.

[1] P. Jönsson, G. Gaigalas, J. Bieron, C. Froese Fischer, I. Grant, Comput. Phys. Commun. (2012) submitted.

[2] E. Gaidamauskas, C. Nazé, P. Rynkun, G. Gaigalas, P. Jönsson, M. Godefroid, J. Phys. B: At. Mol. Opt. Phys. 44 (17) (2011) 175003.

[3] P. Jönsson, C. Froese Fischer, Comput. Phys. Commun. 100 (1997) 81-92.

\section{Introduction}

When the effects of the finite mass of the nucleus and the spatial charge distribution are taken into account in a Hamiltonian describing an atomic system, the isotopes of an element have different electronic energy levels. The isotope shift (IS) is formed by two contributions: the mass shift (MS) and the field shift (FS). The field shift, caused by the penetration of the electron wave functions into the nuclear region, dominates for heavy atoms whereas the mass shift plays a prominent role for light atoms. Relativistic effects are expected to increase with the nuclear charge while the mass shift contribution should become less significant. Shabaev and Artemyev [6] and, in an independent way, Palmer [7], derived these relativistic corrections to the mass shift. Since then several papers have shown the importance of such relativistic effects to the isotope shift [1, 2, 3, 4, 5].

The present relativistic isotope shift program (RIS3), designed as a module of GRASP2K version 1_1 multiconfiguration Dirac-Fock package [8], is to a large extent based on the tensorial expressions derived by Gaidamauskas et al. [9], and we refer to this paper for a more comprehensive theoretical treatment. 


\section{Installation of the program}

As the first step, go to the main directory grasp2K_v1_1 of the new GRASP2K package. Type source make-environment_xxx where $\mathrm{xxx}$ is the compiler name (see README file of the GRASP2K package). The GRASP2K environment variables are now set. Prepare backup copies of the Makefiles in grasp2K_v1_1/src/ and in grasp2K_v1_1/src/appl/v3. These two Makefiles will be modified during the installation of the RIS3 program so that it becomes a fully integrated part of the GRASP2K package. If anything goes wrong during the installation process the user can always revert to the original Makefiles.

To install the RIS3 programs, copy the file RIS3_main.tgz to the main directory grasp2K_v1_1 of the new release of the GRASP2K package. Untar by typing tar -zxvf RIS3 main.tgz. A directory RIS3 main will appear as well as a script Install_RIS3. In the grasp2K_v1_1 directory, execute the installation script by typing ./Install_RIS3. This will generate the executables ris3 and ris3_ljs of the RIS3 program package without compromising the other binaries. The executables are automatically moved to the subdirectory grasp2K_v1_1/bin. The names of the executables follow the conventions in GRASP2K, where the extension 3 indicates that the angular part of the programs are based on a combination of second quantization in the coupled tensorial form, angular momentum theory in three spaces (orbital, spin and quasispin), and a generalized graphical technique [10, 11].

Please note that the command source make-environment_xxx must be issued before any installation of RIS3, since the environment variable \$GRASP needs to be defined.

\section{Theory}

\subsection{Isotope shifts and sign convention}

The frequency of a spectral line $k$ connecting levels $\ell \leftrightarrow u$, with $E_{u}>E_{\ell}$,

$$
\nu_{k}=\frac{E_{u}-E_{\ell}}{h},
$$

differs from one isotope to another. The line frequency isotope shift between isotopes $A$ and $A^{\prime}$

$$
\delta \nu_{k}^{A, A^{\prime}} \equiv \nu_{k}^{A}-\nu_{k}^{A^{\prime}}=\frac{\delta E_{u}^{A, A^{\prime}}-\delta E_{\ell}^{A, A^{\prime}}}{h},
$$


with

$$
\delta E_{i}^{A, A^{\prime}}=E_{i}^{A}-E_{i}^{A^{\prime}} \quad(i=\ell, u),
$$

is said to be "normal" if the line frequency is higher for the heaviest isotope, i.e. if $\delta \nu^{A, A^{\prime}}>0$, with $A>A^{\prime}$ [12]. This terminology is consistent with our previous work on IS on electron affinities [13, 14, 15] and is natural when restricting the IS to the "normal" mass shift (see end of section 3.3.

\subsection{Numerical method}

In the muticonfiguration Dirac-Hartree-Fock (MCDHF) method, the atomic state function (ASF), of a state of an atom, is expressed as a linear combination of symmetry-adapted configuration state functions (CSFs), i.e.

$$
\left|\Psi_{P J M_{J}}\right\rangle=\sum_{\nu=1} c_{\nu}\left|\Phi\left(\gamma_{\nu} P J M_{J}\right)\right\rangle
$$

where $\gamma_{\nu}, P, J$ and $M_{J}$ denote, respectively, the configuration and its coupling tree, the parity, the total angular quantum number and its projection on the $z$-axis $J_{z}$.

The numerical MCDHF method is a variational method in which the radial parts of the one-electron orbitals, used for building the CSFs, and the mixing coefficients $c_{\nu}$ are obtained in a self consistent procedure that optimizes the energy functional

$$
E=\sum_{\mu=1} \sum_{\nu=1} c_{\mu} c_{\nu}\left\langle\Phi\left(\gamma_{\mu} P J M_{J}\right)\left|H_{D C}\right| \Phi\left(\gamma_{\nu} P J M_{J}\right)\right\rangle .
$$

The latter is estimated from the expectation value of the Dirac-Coulomb Hamiltonian [16]

$$
H_{D C}=\sum_{i=1}^{N}\left(c \boldsymbol{\alpha}_{i} \cdot \mathbf{p}_{i}+\left(\beta_{i}-1\right) c^{2}+V\left(r_{i}\right)\right)+\sum_{i<j}^{N} \frac{1}{r_{i j}}
$$

where $V\left(r_{i}\right)$ is the monopole part of the electron-nucleus interaction, $\boldsymbol{\alpha}$ and $\beta$ are the $(4 \times 4)$ Dirac matrices and $c$ is the speed of light $(c=1 / \alpha$ in atomic units, where $\alpha$ is the fine-structure constant). 


\subsection{Mass shift}

The nuclear recoil corrections within the $(\alpha Z)^{4} m^{2} / M$ approximation are obtained by evaluating the expectation values of the operator [6, 1]

$$
H_{\mathrm{MS}}=\frac{1}{2 M} \sum_{i, j}^{N}\left(\mathbf{p}_{i} \cdot \mathbf{p}_{j}-\frac{\alpha Z}{r_{i}}\left(\boldsymbol{\alpha}_{i}+\frac{\left(\boldsymbol{\alpha}_{i} \cdot \mathbf{r}_{i}\right) \mathbf{r}_{i}}{r_{i}^{2}}\right) \cdot \mathbf{p}_{j}\right),
$$

with the Dirac wave functions, eigenfunctions of the operator (6). Rewriting (7) as the sum of the NMS and SMS contributions, i.e. separating the

one-body and two-body terms, and using the tensorial form $\mathbf{r}^{1}=r \mathbf{C}^{1}$, (7) becomes

$$
H_{\mathrm{MS}}=H_{\mathrm{NMS}}+H_{\mathrm{SMS}}
$$

with

$$
\begin{aligned}
H_{\mathrm{NMS}} & =\frac{1}{2 M} \sum_{i=1}^{N}\left(\mathbf{p}_{i}^{2}-\frac{\alpha Z}{r_{i}} \boldsymbol{\alpha}_{i} \cdot \mathbf{p}_{i}-\frac{\alpha Z}{r_{i}}\left(\boldsymbol{\alpha}_{i} \cdot \mathbf{C}_{i}^{1}\right) \mathbf{C}_{i}^{1} \cdot \mathbf{p}_{i}\right) \\
H_{\mathrm{SMS}} & =\frac{1}{2 M} \sum_{i \neq j}^{N}\left(\mathbf{p}_{i} \cdot \mathbf{p}_{j}-\frac{\alpha Z}{r_{i}} \boldsymbol{\alpha}_{i} \cdot \mathbf{p}_{j}-\frac{\alpha Z}{r_{i}}\left(\boldsymbol{\alpha}_{i} \cdot \mathbf{C}_{i}^{1}\right) \mathbf{C}_{i}^{1} \cdot \mathbf{p}_{j}\right)
\end{aligned}
$$

that, in both cases, are rewritten as sums of three separate contributions:

$$
H_{\mathrm{NMS}} \equiv H_{\mathrm{NMS}}^{1}+H_{\mathrm{NMS}}^{2}+H_{\mathrm{NMS}}^{3}
$$

and

$$
H_{\mathrm{SMS}} \equiv H_{\mathrm{SMS}}^{1}+H_{\mathrm{SMS}}^{2}+H_{\mathrm{SMS}}^{3} \cdot
$$

The (mass-independent) normal mass shift $K_{\mathrm{NMS}}$ and specific mass shift $K_{\mathrm{SMS}}$ parameters are defined by the following expressions

$$
\begin{aligned}
\frac{K_{\mathrm{NMS}}}{M} & \equiv\left\langle\Psi\left(\gamma P J M_{J}\right)\left|H_{\mathrm{NMS}}\right| \Psi\left(\gamma P J M_{J}\right)\right\rangle \\
& =\left[\Psi(\gamma P J)\left\|H_{\mathrm{NMS}}\right\| \Psi(\gamma P J)\right], \\
\frac{K_{\mathrm{SMS}}}{M} & \equiv\left\langle\Psi\left(\gamma P J M_{J}\right)\left|H_{\mathrm{SMS}}\right| \Psi\left(\gamma P J M_{J}\right)\right\rangle \\
& =\left[\Psi(\gamma P J)\left\|H_{\mathrm{SMS}}\right\| \Psi(\gamma P J)\right],
\end{aligned}
$$


where each second equality gives, thanks to Wigner-Eckart theorem [17], the relation between the matrix element of the mass shift operators and their reduced matrix element (r.m.e.). By analogy with (11) and (12), these mean values can be expressed as $K_{\mathrm{NMS}}=K_{\mathrm{NMS}}^{1}+K_{\mathrm{NMS}}^{2}+K_{\mathrm{NMS}}^{3}$ and $K_{\mathrm{SMS}}=$ $K_{\mathrm{SMS}}^{1}+K_{\mathrm{SMS}}^{2}+K_{\mathrm{SMS}}^{3}$. The $K^{1}$ term is generally labeled the "uncorrected" value and the sum " $K^{2}+K^{3}$ " represents its "corrections".

It is important to notice that the program SMS92 [18], the first relativistic isotope shift program designed for GRASP92 [19], calculates the NMS as the expectation value $\left\langle\sum_{i} T_{i}\right\rangle$, where $T_{i}$ is the Dirac kinetic energy operator $T_{i}=c \boldsymbol{\alpha}_{i} \cdot \mathbf{p}_{i}+\left(\beta_{i}-1\right) c^{2}$ associated with electron $i$, while the program RIS3 uses the expectation value $\left\langle H_{\mathrm{NMS}}^{1}\right\rangle=\left\langle\sum_{i} p_{i}^{2} / 2 M\right\rangle$ built on the relativistic electron momenta. This issue is discussed in [20].

When discussing the transition isotope shift (2), one needs to consider the variation of the mass parameter from one level to another. The corresponding line frequency isotope mass shift is written as the sum of the normal mass shift (NMS) and specific mass shift (SMS) contributions:

$$
\delta \nu_{k, \mathrm{MS}}^{A, A^{\prime}}=\delta \nu_{k, \mathrm{NMS}}^{A, A^{\prime}}+\delta \nu_{k, \mathrm{SMS}}^{A, A^{\prime}}
$$

with

$$
\delta \nu_{k, \mathrm{MS}}^{A, A^{\prime}}=\left(\frac{M^{\prime}-M}{M M^{\prime}}\right) \frac{\Delta K_{\mathrm{MS}}}{h}=\left(\frac{M^{\prime}-M}{M M^{\prime}}\right) \Delta \widetilde{K}_{\mathrm{MS}}
$$

where $\Delta K_{\mathrm{MS}}=\left(K_{\mathrm{MS}}^{u}-K_{\mathrm{MS}}^{\ell}\right)$ is the difference of the $K_{\mathrm{MS}}\left(=K_{\mathrm{NMS}}+K_{\mathrm{SMS}}\right)$ parameters of the levels involved in transition $k$. As far as conversion factors are concerned, we use ${ }^{2} \Delta K_{\mathrm{MS}}\left[m_{e} E_{\mathrm{h}}\right]=3609.4824 \Delta \widetilde{K}_{\mathrm{MS}}[\mathrm{GHz} u]$. Note that thanks to (8), equation (16) applies separately to both the NMS and SMS mass contributions. When applied to the normal mass shift, the line frequency isotope shift $\delta \nu_{k, \mathrm{NMS}}^{A, A^{\prime}}\left(A>A^{\prime}\right)$ is a positive quantity since both factors $\left(M^{\prime}-M\right)$ and $\Delta K_{\mathrm{NMS}}=\left(K_{\mathrm{NMS}}^{u}-K_{\mathrm{NMS}}^{\ell}\right)$ are necessarily negative. This observation is at the origin of the terminology qualifying a positive frequency shift as a "normal" one, corresponding to a line shift toward larger wavenumbers for the heavier isotope.

\footnotetext{
${ }^{1}$ This convenient notation is related to a more common one through $\left\langle\gamma J^{\prime}\left\|O^{k}\right\| \gamma J\right\rangle=$ $\sqrt{2 J^{\prime}+1}\left[\gamma J^{\prime}\|O\| \gamma J\right]$.

${ }^{2}$ This conversion factor is calculated as $\left(m_{e} / u\right) 2 R_{\infty} c \times 1.10^{-9}=3609.4824$ using the 2006 CODATA recommended values of the fundamental physical constants [21].
} 


\subsection{Field shift theory}

The energy shift arising from the difference in nuclear charge distributions between two isotopes $A$ and $A^{\prime}$ for a given atomic state $(i)$ is called the level field shift (FS). It can be estimated from the difference of the total energies that are obtained, for level $(i)$, by solving separately the infinite mass MCDHF equations, using realistic nuclear charge distributions for isotopes $\left(A, A^{\prime}\right)$

$$
\delta E_{i}^{A, A^{\prime}}=E_{i}^{A}-E_{i}^{A^{\prime}} .
$$

When considering a wide range of different isotopes, 17 becomes inconvenient since the electronic wave functions have to be determined for all the isotopes considered. Adopting the electronic density $\rho_{i, A^{\prime}}^{e}$ for the zeroth-order picture, perturbation theory can be used instead to get the first-order energy correction [22, 23]

$$
\delta E_{i}^{(1) A, A^{\prime}}=-\frac{e^{2}}{4 \pi \epsilon_{0}} \iint d \mathbf{r} d \mathbf{r}^{\prime} \frac{1}{\left|\mathbf{r}-\mathbf{r}^{\prime}\right|} \rho_{i, A^{\prime}}^{e}(\mathbf{r})\left[\rho_{A}^{N}\left(\mathbf{r}^{\prime}\right)-\rho_{A^{\prime}}^{N}\left(\mathbf{r}^{\prime}\right)\right],
$$

where $\rho^{N}$ and $\rho_{i}^{e}$ are respectively, the nuclear charge density and level electronic density of a given isotope.

Assuming a spherical nuclear charge distribution and expanding the electron density within the nucleus $\left(r<R_{\text {nucl }}\right)$ as a polynomial

$$
\rho^{e}(r)=b_{0}+b_{2} r^{2}+b_{4} r^{4}+\ldots,
$$

the level field shift (18) can be written as [22, 23]

$$
\delta E_{i}^{(1) A, A^{\prime}}=\mathcal{F}_{i} \lambda^{A, A^{\prime}}
$$

where

$$
\lambda^{A, A^{\prime}}=\delta\left\langle r^{2}\right\rangle^{A, A^{\prime}}+\frac{C_{2}}{C_{1}} \delta\left\langle r^{4}\right\rangle^{A, A^{\prime}}+\frac{C_{3}}{C_{1}} \delta\left\langle r^{6}\right\rangle^{A, A^{\prime}}+\ldots
$$

is the so-called "nuclear parameter" originally introduced by Seltzer [24], reflecting the isotopic variation of the nuclear charge distribution, and where $\mathcal{F}_{i}$ is the level electronic factor

$$
\mathcal{F}_{i}=\frac{2 \pi}{3} Z\left(\frac{e^{2}}{4 \pi \epsilon_{0}}\right)|\Psi(0)|_{i}^{2} .
$$


In the latter expression, $|\Psi(0)|^{2}$ is the total probability density at the origin that can be estimated by taking the $\mathbf{r} \rightarrow \mathbf{0}$ limit of the electron density [25]

$$
|\Psi(0)|_{i}^{2}=\lim _{\mathbf{r} \rightarrow \mathbf{0}} \rho_{i, A^{\prime}}^{e}(\mathbf{r})=\frac{1}{4 \pi} \lim _{r \rightarrow 0} \rho_{i, A^{\prime}}^{e}(r),
$$

using the electron wave function calculated for the reference isotope $A^{\prime}$.

Using (20) for levels $i=(\ell, u)$ involved in transition $k$, the frequency field shift of the spectral line $k$ can be written as [12, 22, 23]

$$
\delta \nu_{k, \mathrm{FS}}^{A, A^{\prime}}=\frac{\delta E_{u}^{(1) A, A^{\prime}}-\delta E_{\ell}^{(1) A, A^{\prime}}}{h}=F_{k} \lambda^{A, A^{\prime}}
$$

where $F_{k}$ is the line electronic factor

$$
F_{k}=\frac{2 \pi}{3 h} Z\left(\frac{e^{2}}{4 \pi \epsilon_{0}}\right) \Delta|\Psi(0)|_{k}^{2},
$$

proportional to the change of the total probability density at the origin

$$
\Delta|\Psi(0)|_{k}^{2}=\Delta \rho_{k}^{e}(\mathbf{0})=\rho_{u}^{e}(\mathbf{0})-\rho_{\ell}^{e}(\mathbf{0})
$$

associated with the electronic transition between levels $\ell$ and $u$. The merit of (20) and (24) is to factorize the FS into the electronic factors $\mathcal{F}$ and $F$ depending on the atomic state, and the nuclear parameter $\lambda^{A, A^{\prime}}$.

If the first term in Eq. 21] is dominant, the higher order contributions can be neglected. In this approximation, the first-order level- and frequencyfield shifts (20) and (24) become

$$
\delta E_{i}^{(1) A, A^{\prime}} \simeq \frac{2 \pi}{3} Z\left(\frac{e^{2}}{4 \pi \epsilon_{0}}\right)\left|\Psi_{A^{\prime}}(0)\right|_{i}^{2} \delta\left\langle r^{2}\right\rangle^{A, A^{\prime}},
$$

and

$$
\delta \nu_{k, F S}^{A, A^{\prime}} \simeq \frac{Z}{3 \hbar}\left(\frac{e^{2}}{4 \pi \epsilon_{0}}\right) \Delta\left|\Psi_{A^{\prime}}(0)\right|_{k}^{2} \delta\left\langle r^{2}\right\rangle^{A, A^{\prime}}
$$

with

$$
\delta\left\langle r^{2}\right\rangle^{A, A^{\prime}}=\left\langle r^{2}\right\rangle^{A}-\left\langle r^{2}\right\rangle^{A^{\prime}}
$$

Let us take $A>A^{\prime}$. If the nuclear charge distribution expands with the number of nucleons $\left(\delta\left\langle r^{2}\right\rangle^{A, A^{\prime}}>0\right)$, the transition field shift frequency is negative $\left(\delta \nu_{k, F S}^{A, A^{\prime}}<0\right)$ when the upper level $(u)$ has an electron density at the origin smaller than the one of the lower level $(\ell)$, i.e. $\Delta\left|\Psi_{A^{\prime}}(0)\right|_{k}^{2}<0$. In this case, the FS contribution to the line isotope shift could be qualified as "anomalous" in the sense that it counteracts the "normal" mass shift. 


\section{Outline of the method}

Using multiconfiguration expansions (4), the reduced matrix elements of a general spherical tensor operator $T_{q}^{k}$ becomes [16]

$$
\left[\Psi(\gamma P J)\left\|\mathbf{T}^{k}\right\| \Psi(\gamma P J)\right]=\sum_{\mu, \nu} c_{\mu} c_{\nu}\left[\Phi\left(\gamma_{\mu} P J\right)\left\|\mathbf{T}^{k}\right\| \Phi\left(\gamma_{\nu} P J\right)\right]
$$

\subsection{Expectation value of one-body operators}

\subsubsection{The normal mass shift}

The reduced matrix elements of the one-electron operator $H_{\mathrm{NMS}}=\sum_{i} h_{\mathrm{NMS}}(i)$ between CSFs is expressed as a sum over single-particle reduced matrix elements [16]

$$
\left[\Phi\left(\gamma_{\mu} P J\right)\left\|H_{\mathrm{NMS}}\right\| \Phi\left(\gamma_{\nu} P J\right)\right]=\sum_{a, b} t_{\mu \nu}^{0}(a b)\left[n_{a} \kappa_{a}\left\|h_{\mathrm{NMS}}\right\| n_{b} \kappa_{b}\right]
$$

where the $t_{\mu \nu}^{0}(a b)$ are the pure spin-angular coefficients arising from Racah's algebra [26, 27]

$$
\begin{aligned}
& t_{\mu \nu}^{0}(a b)=(-1)^{\Delta+1} \sqrt{2 j_{a}+1} R\left(j_{a}, j_{b}, \Lambda_{\mu}, \Lambda_{\nu}\right) \delta\left(\kappa_{a}, \kappa_{b}\right) \\
\times & \left\{\delta\left(n_{a}, n_{b}\right)\left(j_{a}^{N_{a}} \alpha_{a} Q_{a} J_{a}\left\|\left[a_{\frac{1}{2}}^{\left(q j_{a}\right)} \times a_{-\frac{1}{2}}^{\left(q j_{a}\right)}\right]^{(0)}\right\| j_{a}^{N_{a}} \alpha_{a} Q_{a} J_{a}\right)\right. \\
+ & \left(1-\delta\left(n_{a}, n_{b}\right)\right)\left(j_{a}^{N_{a}} \alpha_{a} Q_{a} J_{a}\left\|a_{\frac{1}{2}}^{\left(q j_{a}\right)}\right\| j_{a}^{N_{a}^{\prime}} \alpha_{a} Q_{a} J_{a}\right) \\
\times & \left.\left(j_{b}^{N_{b}} \alpha_{b} Q_{b} J_{b}\left\|a_{-\frac{1}{2}}^{\left(q j_{b}\right)}\right\| j_{b}^{N_{b}^{\prime}} \alpha_{b} Q_{b} J_{b}\right)\right\} .
\end{aligned}
$$

The recoupling matrix $R\left(j_{a}, j_{b}, \Lambda_{\mu}, \Lambda_{\nu}\right)$ is either a product of delta functions [27, eq. (18)] when $n_{a} \kappa_{a}=n_{b} \kappa_{b}$ or a combination of delta functions and $6 j$-coefficients [27, eq. (22)] when $n_{a} \kappa_{a} \neq n_{b} \kappa_{b}$. The symbols $\Lambda_{\mu} \equiv$ $\left(J_{a}, J_{b}, J_{a^{\prime}}, J_{b^{\prime}}\right)_{\mu}$ and $\Lambda_{\nu} \equiv\left(J_{a}, J_{b}, J_{a^{\prime}}, J_{b^{\prime}}\right)_{\nu}$ denote the respective sets of active subshell angular momenta. The operators $a_{m_{q}}^{(q j)}$ are second quantization operators in quasispin space of rank $q=\frac{1}{2}$. The operator $a_{\frac{1}{2} m_{j}}^{(q j)}=a_{m_{j}}^{(j)}$ creates electrons with angular momentum quantum numbers $j, m_{j}$ and its conjugate $a_{-\frac{1}{2} m_{j}}^{(q j)}=\tilde{a}_{m_{j}}^{(j)}=(-1)^{j-m_{j}} a_{-m_{j}}^{(j)+}$ annihilates electrons with the same quantum numbers $j, m_{j}$ in a given subshell. 
The phase factor in 32 arises from the reordering needed to match the recoupled creation and annihilation operators in the bra and ket vectors contributing to the matrix element. We have $\Delta=0$ when $n_{a} \kappa_{a}=n_{b} \kappa_{b}$; otherwise its value is given by

$$
\Delta=1+\sum_{r=i}^{j-1} N_{r}
$$

where $N_{r}$ is the occupation number of a subshell $r, i=\min \{a, b\}$ and $j=$ $\max \{a, b\}$.

The radial part of the normal mass shift operator is given by

$$
\begin{aligned}
& {\left[n_{a} \kappa_{a}\left\|h_{\mathrm{NMS}}\right\| n_{b} \kappa_{b}\right]=\delta\left(\kappa_{a}, \kappa_{b}\right) } \\
\times & \frac{1}{2 M} \int_{0}^{\infty}\left(\left(\partial_{r} P_{a}\right)\left(\partial_{r} P_{b}\right)+\left(\partial_{r} Q_{a}\right)\left(\partial_{r} Q_{b}\right)+\frac{l_{b}\left(l_{b}+1\right) P_{a} P_{b}+\widetilde{l}_{b}\left(\widetilde{l}_{b}+1\right) Q_{a} Q_{b}}{r^{2}}\right. \\
+ & \left.(-2 \alpha Z) \frac{Q_{a} \partial_{r} P_{b}+Q_{b} \partial_{r} P_{a}}{r}+(-\alpha Z)\left(\frac{\kappa_{b}-1}{r^{2}}\right)\left(Q_{a} P_{b}+Q_{b} P_{a}\right)\right) d r .
\end{aligned}
$$

We used the notations $\tilde{l}=2 j-l$ and $\partial_{r} \equiv \frac{\partial}{\partial r}$. $P_{a}$ and $Q_{a}$ are respectively the large and small components of the relativistic one-electron radial wave function $a=\left(n_{a} \kappa_{a}\right)$, where $\kappa=(l-j)(2 j+1)$.

\subsubsection{The field shift}

The electron density at the origin, needed to calculate the FS in the approximation (27), is also the mean value of a one-body operator:

$$
\rho^{e}(\mathbf{r})=\sum_{\mu=1} \sum_{\nu=1} c_{\mu} c_{\nu}\left\langle\Phi\left(\gamma_{\mu} \Pi J M_{J}\right)|\delta(\mathbf{r})| \Phi\left(\gamma_{\nu} \Pi J M_{J}\right)\right\rangle
$$

where $\delta(\mathbf{r})$ probes the presence of electrons at a particular point in space, here at the origin, and can be written as the one-electron first-quantization operator $\delta(\mathbf{r})=\sum_{i=1}^{N} \delta\left(\mathbf{r}-\mathbf{r}_{i}\right)$. The reduction to sums over radial integral is strictly equivalent to the one of the NMS

$$
\left\langle\phi\left(\gamma_{\mu} P J M_{J}\right)\left|\sum_{i=1}^{N} \delta\left(\mathbf{r}_{i}\right)\right| \phi\left(\gamma_{\nu} P J M_{J}\right)\right\rangle=\sum_{a, b} t_{\mu \nu}^{0}(a b) I_{\mathrm{FS}}(a b),
$$


where $\delta\left(\mathbf{r}_{i}\right)$ probes the presence of electron $i$ at the origin, $t_{\mu \nu}^{0}(a b)$ are the pure spin-angular coefficient (32) and $I_{\mathrm{FS}}(a b)$, in the relativistic multiconfiguration approximation, is expressed as [18, 28]

$$
\left.\left(\frac{P_{n \kappa}(r) P_{n^{\prime} \kappa}(r)+Q_{n \kappa}(r) Q_{n^{\prime} \kappa}(r)}{4 \pi r^{2}}\right)\right|_{r \rightarrow 0} .
$$

\subsection{Expectation value of the two-body operator}

Using Racah's algebra one can express the r.m.e of any two-particle operator as [26, 27]

$$
\begin{aligned}
& {\left[\Phi\left(\gamma_{\mu} P J\right)\left\|\sum_{i<j} g(i, j)\right\| \Phi\left(\gamma_{\nu} P J\right)\right] } \\
= & \sum_{a b c d} \sum_{k_{12}, \sigma_{12}, k_{12}^{\prime}, \sigma_{12}^{\prime}} \sum_{K_{l} K_{s}}(-1)^{\Delta} \Theta^{\prime}\left(n_{a} l_{a} j_{a}, n_{b} l_{b} j_{b}, n_{c} l_{c} j_{c}, n_{d} l_{d} j_{d}, \Xi\right) \\
\times & T\left(n_{a} j_{a}, n_{b} j_{b}, n_{c} j_{c}, n_{d} j_{d}, \Lambda_{\mu}, \Lambda_{\nu}, \Xi, \Gamma\right) R\left(j_{a}, j_{b}, j_{c}, j_{d}, \Lambda_{\mu}, \Lambda_{\nu}, \Gamma\right) .
\end{aligned}
$$

The two last sums are over intermediate ranks $k_{12}, \sigma_{12}, k_{12}^{\prime}, \sigma_{12}^{\prime}, K_{l}$ and $K_{s}$ appearing in the expression of $T\left(n_{a} j_{a}, n_{b} j_{b}, n_{c} j_{c}, n_{d} j_{d}, \Lambda_{\mu}, \Lambda_{\nu}, \Xi, \Gamma\right)$.

As for the one-body operator, the phase factors $\Delta$ arise from the reordering necessary to match the recoupled creation and annihilation operators in bra and ket vectors. $\Gamma$ specifies the recoupling scheme for each matrix element and $\Xi$, when required, specifies the coupling scheme of the tensor operators defining each matrix element.

The term $R\left(j_{a}, j_{b}, j_{c}, j_{d}, \Lambda_{\mu}, \Lambda_{\nu}, \Gamma\right)$ is the recoupling coefficient. In the case of one interacting shell it reduces to delta functions [27, eq. (18)]. For two, three and four interacting shells, the recoupling coefficients are given by [27, eqs. (22), (26) and (33)], replacing $l, L$ by $j, J$ respectively.

The terms $T\left(n_{a} j_{a}, n_{b} j_{b}, n_{c} j_{c}, n_{d} j_{d}, \Lambda_{\mu}, \Lambda_{\nu}, \Xi, \Gamma\right)$ in (38) are reduced matrix elements of standard subshell creation/annihilation operators

$$
\begin{aligned}
a & =a_{m_{q}}^{(q j)} \\
W & =\left[a_{m_{q 1}}^{(q j)} \times a_{m_{q 2}}^{(q j)}\right]^{\left(k_{1} \sigma_{1}\right)}, \\
a W & =\left[a_{m_{q 1}}^{(q j)} \times\left[a_{m_{q 2}}^{(q j)} \times a_{m_{q 3}}^{(q j)}\right]^{\left(k_{1} \sigma_{1}\right)}\right]^{\left(k_{2} \sigma_{2}\right)},
\end{aligned}
$$




$$
\begin{aligned}
W a & =\left[\left[a_{m_{q 1}}^{(q j)} \times a_{m_{q 2}}^{(q j)}\right]^{\left(k_{1} \sigma_{1}\right)} \times a_{m_{q 3}}^{(q j)}\right]^{\left(k_{2} \sigma_{2}\right)}, \\
W W & =\left[\left[a_{m_{q 1}}^{(q j)} \times a_{m_{q 2}}^{(q j)}\right]^{\left(k_{1} \sigma_{1}\right)} \times\left[a_{m_{q 3}}^{(q j)} \times a_{m_{q 4}}^{(q j)}\right]^{\left(k_{2} \sigma_{2}\right)}\right]^{(k k)} .
\end{aligned}
$$

The creation and annihilation operators in (39)- 43 refer to a single subshell. The evaluation of the submatrix elements of these operators is explained in [27].

This general expression is implemented in GRASP2K version 1_1, but what is needed for the SMS evaluation is much simpler, since $k_{1}=k_{2}=1$, $\sigma_{1}=\sigma_{2}=0$ and $k=0$, such as all three terms of $(10)$ have the particular form

$$
g(i, j)=g_{1}\left(r_{i}\right) g_{1}\left(r_{j}\right)\left(\mathbf{T}^{1}(i) \cdot \mathbf{T}^{1}(j)\right) .
$$

The reduction of the many-electron r.m.e. can be done in terms of twoelectron integrals $X^{1}$, also called effective interaction strengths [16],

$$
\left[\Phi\left(\gamma_{\mu} P J\right)\left\|\sum_{i<j} g(i, j)\right\| \Phi\left(\gamma_{\nu} P J\right)\right]=\sum_{a b c d} v_{\mu \nu}^{(1)}(a b c d) X^{1}(a b c d)
$$

Adopting the covariant notation for the $3 j$-symbol of Wigner [29] and using the definition of the scalar product of two irreducible tensor operators and the Wigner-Eckart theorem, the matrix element of (44) can be written as follows

$$
\langle a b|g(i, j)| c d\rangle=\sum_{q=-1}^{1}\left(\begin{array}{ccc}
m_{a} & 1 & j_{c} \\
j_{a} & q & m_{c}
\end{array}\right)\left(\begin{array}{ccc}
m_{b} & q & j_{d} \\
j_{b} & 1 & m_{d}
\end{array}\right) X^{1}(a b c d)
$$

where

$$
X^{1}(a b c d)=-\left\langle a\left\|g\left(r_{i}\right) \mathbf{T}^{1}(i)\right\| c\right\rangle\left\langle b\left\|g\left(r_{j}\right) \mathbf{T}^{1}(j)\right\| d\right\rangle .
$$

From the structure of $(12)$, the latter has three components

$$
X^{1}(a b c d)=X_{1}^{1}(a b c d)+X_{2}^{1}(a b c d)+X_{3}^{1}(a b c d),
$$

that have the from

$$
X_{1}^{1}(a b c d)=\left\langle\kappa_{a}\left\|\mathbf{C}^{1}\right\| \kappa_{c}\right\rangle\left\langle\kappa_{b}\left\|\mathbf{C}^{1}\right\| \kappa_{d}\right\rangle \mathcal{V}\left(n_{a} \kappa_{a}, n_{c} \kappa_{c}\right) \mathcal{V}\left(n_{b} \kappa_{b}, n_{d} \kappa_{d}\right),
$$




$$
\begin{gathered}
X_{2}^{1}(a b c d)=-\left\langle\kappa_{b}\left\|\mathbf{C}^{1}\right\| \kappa_{d}\right\rangle \mathcal{V}\left(n_{b} \kappa_{b}, n_{d} \kappa_{d}\right) \\
\times \quad \int_{0}^{\infty} d r\left(\frac{-\alpha Z}{r}\right)\left(-Q_{a} P_{c}\left\langle-\kappa_{a}\left\|\boldsymbol{\sigma}^{1}\right\| \kappa_{c}\right\rangle+Q_{c} P_{a}\left\langle\kappa_{a}\left\|\boldsymbol{\sigma}^{1}\right\|-\kappa_{c}\right\rangle\right)
\end{gathered}
$$

and

$$
\begin{aligned}
X_{3}^{1}(a b c d)= & -\left\langle\kappa_{b}\left\|\mathbf{C}^{1}\right\| \kappa_{d}\right\rangle\left\langle\kappa_{a}\left\|\mathbf{C}^{1}\right\| \kappa_{c}\right\rangle \mathcal{V}\left(n_{b} \kappa_{b}, n_{d} \kappa_{d}\right) \\
& \times \int_{0}^{\infty} d r\left(\frac{-\alpha Z}{r}\right)\left(Q_{a} P_{c}-Q_{c} P_{a}\right),
\end{aligned}
$$

where $\mathcal{V}\left(n \kappa, n^{\prime} \kappa^{\prime}\right)$ is a Vinti relativistic integral. Its analytic expression as well as the ones of the three reduced angular one-electron matrix elements $\left\langle\kappa\left\|\mathbf{C}^{1}\right\| \kappa^{\prime}\right\rangle,\left\langle-\kappa\left\|\boldsymbol{\sigma}^{1}\right\| \kappa^{\prime}\right\rangle$ and $\left\langle\kappa\left\|\boldsymbol{\sigma}^{1}\right\|-\kappa^{\prime}\right\rangle$ can be found in [9].

\section{Program structure}

The program SMS92 [18], predecessor of this version, was designed to be used in two different ways: -i) if the electronic wave function was obtained from a relativistic self-consistent process run, the angular coefficients could be reused. -ii) if the wave function resulted from a relativistic configurationinteraction (RCI) run, the program calculated angular coefficients as they were needed. This procedure has been replaced by the possibility of storing angular coefficients in files. If requested by the user, the one-body angular coefficients associated to $K_{\mathrm{NMS}}$ and the density are stored in a file with extension . IOB while the two-body ones, corresponding to $K_{\mathrm{SMS}}$, are stored in a file with extension . ITB. These two files can be reused in later calculations, saving a considerable amount of CPU time. If only one of the angular file is present, the program will adapt itself and ask if the other one must be saved.

\subsection{Files naming conventions}

The passing of information between the RIS3 program and other programs of the GRASP2K version 1_1 package is done through files. This process is greatly facilitated through file naming conventions. A name is associated with the files that define the wave function and holds the output of the different calculations. An extension defines the contents and format of the file. Thus the file name becomes name.extension. The files associated with the RIS3 program are given below: 


$\begin{array}{ll}\text { name.c } & \text { Configuration state list } \\ \text { name.w } & \text { Binary file of radial functions } \\ \text { name.m } & \begin{array}{l}\text { Binary file of expansion or mixing } \\ \text { coefficients produced by rscf or its variants }\end{array} \\ \text { name.cm } & \begin{array}{l}\text { Binary file of mixing coefficients produced by rci } \\ \text { or its variants }\end{array} \\ \text { name.lsj.lbl } & \begin{array}{l}\text { File giving the } L S J \text { expansion information of } \\ \text { the wave functions }\end{array} \\ \text { name.i } & \text { Isotope shift data obtained with rscf mixing coefficients } \\ \text { name.ci } & \text { Isotope shift data obtained with rci mixing coefficients } \\ \text { name.IOB } & \text { File containing one-body angular coefficients } \\ \text { name.ITB } & \text { File containing two-body angular coefficients }\end{array}$

\subsection{Additional tool}

JJ2LSJ is an additional program of GRASP2K version 1_1 package that allows a transformation of ASFs from a $j j$-coupled CSF basis into an $L S J$ coupled CSF basis. This useful tool has been adapted to the isotope shift output: RIS_LSJ. It prints output from the RIS3 program using $L S J$ labels. The program requires that a name.1sj.1bl file is available along with the name.i or name.ci files. The output can be energy sorted.

RISOSUPPORT integrated in the toolkit, evaluates the level and transition isotope shifts for a given spectral line, for all the isotopes specified by the user. The input data are the $K_{\mathrm{NMS}}, K_{\mathrm{SMS}}$ and $F$ electronic factors (in a.u.), taken from the name. (c) i file, while the atomic masses (from which the nuclear masses are extracted) and root-mean-square nuclear charge radii values are provided by the user. The isotope-shifted transition energies are calculated for all isotopes from the input value given for a reference isotope.

\subsection{New subroutines and functions}

Most of the subroutines used in this program are included in the GRASP2K version $1 \_1$ package [8]. Below we describe only some of the subroutines and functions that are specific for RIS3.

angdata 
checks if name. IOB or/and name. ITB files that contains one or two-body angular coefficients, respectively, do exist.

densmcp

controls the main sequence of routine calls for the calculation of the NMS parameter, the electron density at the origin and some of the radial mean values.

\section{densnew and densread}

are used to, respectively, create or read angular coefficients values in name. IOB file. Then subroutine uses the angular coefficients to build the density, the uncorrected NMS parameter $\left(K_{\mathrm{NMS}}^{1}\right)$, radial mean values $\left(\langle r\rangle,\left\langle r^{2}\right\rangle,\left\langle r^{-1}\right\rangle,\left\langle r^{-2}\right\rangle\right)$ and the sum of NMS parameters $\left(K_{\mathrm{NMS}}^{1}+K_{\mathrm{NMS}}^{2}+K_{\mathrm{NMS}}^{3}\right)$. These are stored in the variables DINT1, DINT2, DINT3, DINT4, DINT5, DINT6, DINT7 respectively.

getmixblock

reads mixing coefficient file from block-structured format.

getsmd

interactively determines the remaining data governing the calculation.

rintdens

given the quantity (37) at a number of grid points close to the nucleus, this subroutine calculates $I_{\mathrm{FS}}(a b)$ by polynomial extrapolation to $r=0$.

rinti_NMS

calculates the r.m.e. $\left[n \kappa\left\|h_{\mathrm{NMS}}\right\| n^{\prime} \kappa^{\prime}\right]$.

rint_sms 2 and rint_sms3

calculate the integrals $X_{2}^{1}(a b c d)$ and $X_{3}^{1}(a b c d)$, respectively.

ris_cal

controls the entire computation.

ris

this routine controls the main sequence of routine calls for the calculation of the MS parameter, the electron density at the origin.

setsum and strsum

initializes the Summary File.

sigma_1 and sigma_2

compute the r.m.e. $\left\langle-\kappa\left\|\boldsymbol{\sigma}^{1}\right\| \kappa^{\prime}\right\rangle$ and $\left\langle\kappa\left\|\boldsymbol{\sigma}^{1}\right\|-\kappa^{\prime}\right\rangle$, respectively.

smsnew and smsread

are use to, respectively, create or read angular coefficients values in .TB file. Then subroutine uses them to build $X_{1}^{1}(a b c d)$ values.

vinti

Calculates the values of Vinti's integrals. 


\section{Input Data}

Several files must be present to run the RIS3 program. The nuclear data needed to construct the radial grid are read from a GRASP2K Isotope Data File (isodata) and three files are required to describe the electronic state:

- a GRASP2K Configuration Symmetry List File (name.c), which lists the subshells, their occupation numbers and the coupling of the subshell total angular moments;

- a GRASP2K Mixing Coefficients file (name.m), which lists the mixing coefficient of each configuration state function in each atomic state function;

- a GRASP2K Radial wave function File (name.w), which stores all radial wave functions on a radial grid;

- the new GRASP2K isotope shifts angular files (name. IOB and/or name. ITB), if available.

In an interactive session, RIS3 asks the user a few questions related to the format and origin of the input files and to the desired output options. A typical session is displayed in Fig. 3 .

By replying, "no", to the default settings, the user may modify the speed of light from its physical value (typically to study non-relativistic limits), change parameters controlling the radial grid, or perform a "first-order" calculation by responding appropriately to prompts. Filenames are provided to the program as responses to prompts. The storage of one- and two-body angular coefficients in files is also left to the user's decision (this option is interesting due to the large amount of memory that two-body angular coefficients can take). If both angular files exist the program will use them and there will be no question about it.

If, for some reason, there are incomplete files with angular coefficients the program will end with some error message when trying to process these files. In these cases the user should remove the files and run the RIS3 program again.

\section{Output Data}

Output data are written to the files name.i or name.ci depending on if the starting wave function was from an rscf or a rci calculation. Following 
a summary of the electronic states and the nuclear parameters, the values of the normal and specific mass shift parameters and electron density at the nucleus are written to the RIS3 output.

For each level considered, the "uncorrected" value $K^{1}$ of NMS or SMS is first presented, followed by the addition of the corrections $K^{2}+K^{3}$ and finally by the sum of the three terms. A first line presents these results in atomic units $\left(E_{\mathrm{h}} m_{e}\right)$, followed by the $\widetilde{K}$ parameters (in $\mathrm{GHz}$ u) defined in $(16)$ ), often used in the literature. In third place comes the value of the density at the origin, in atomic units $\left(a_{0}^{-3}\right)$, for each level.

\section{Acknowledgment}

Cédric Nazé is grateful to the "Fonds pour la formation à la Recherche dans l'Industrie et dans l'Agriculture" of Belgium for a Ph.D. grant (Boursier F.R.S.-FNRS). This work was supported by the Communauté française of Belgium (Action de Recherche Concertée), the Belgian National Fund for Scientific Research (FRFC/IISN Convention) and by the IUAP - Belgian State Science Policy (BriX network P7/12). Per Jönsson gratefully acknowledge financial support from the Swedish Research Council (VR). The authors gratefully acknowledge Dr. Ir. Verdebout for his comments and beta-testing.

[1] I. I. Tupitsyn, V. M. Shabaev, J. R. C. López-Urrutia, I. Draganic, R. S. Orts, J. Ullrich, Relativistic calculations of isotope shifts in highly charged ions, Phys. Rev. A 68 (2003) 022511.

[2] V. A. Korol, M. G. Kozlov, Relativistic corrections to the isotope shift in light ions, Phys. Rev. A 76 (2) (2007) 022103-7.

[3] Y. S. Kozhedub, O. V. Andreev, V. M. Shabaev, I. I. Tupitsyn, C. Brandau, C. Kozhuharov, G. Plunien, T. Stöhlker, Nuclear deformation effect on the binding energies in heavy ions, Phys. Rev. A 77 (2008) 032501.

[4] S. G. Porsev, M. G. Kozlov, D. Reimers, Transition frequency shifts with fine-structure constant variation for Fe I and isotope-shift calculations in Fe I and Fe II, Phys. Rev. A 79 (3) (2009) 032519. doi:10.1103/ PhysRevA.79.032519. 
[5] Y. S. Kozhedub, A. V. Volotka, A. N. Artemyev, D. A. Glazov, G. Plunien, V. M. Shabaev, I. I. Tupitsyn, T. Stöhlker, Relativistic recoil, electron-correlation, and QED effects on the $2 p_{j}-2 s$ transition energies in Li-like ions, Phys. Rev. A 81 (4) (2010) 042513. doi:10.1103/PhysRevA.81.042513.

[6] V. Shabaev, A. Artemyev, Relativistic nuclear recoil corrections to the energy levels of multicharged ions, J. Phys. B : At. Mol. Phys. 27 (1994) $1307-1314$.

[7] C. Palmer, Reformulation of the theory of the mass shift, J. Phys. B : At. Mol. Phys. 20 (1987) 5987-5996.

[8] P. Jönsson, G. Gaigalas, J. Bieron, C. Froese Fischer, I. Grant, Comput. Phys. Commun. (2012) submitted.

[9] E. Gaidamauskas, C. Nazé, P. Rynkun, G. Gaigalas, P. Jönsson, M. Godefroid, Tensorial form and matrix elements of the relativistic nuclear recoil operator, J. Phys. B: At. Mol. Phys. 44 (17) (2011) 175003.

[10] G.A. Gaigalas, Z.B. Rudzikas, and C. Froese Fischer, J. Phys. B: At. Mol. Phys. 30 (1997) 3747.

[11] G. Gaigalas, S. Fritzsche, and Z. Rudzikas, At. Data Nucl. Data Tables 76 (2000) 235.

[12] G. Fricke, C. Bernhardt, K. Heilig, L. Schaller, L. Schellenberg, E. Shera, C. De Jager, Nuclear ground state charge radii from electromagnetic interactions, At. Data Nucl. Data Tables 60 (1995) 177-285.

[13] C. Blondel, C. Delsart, C. Valli, S. Yiou, M. Godefroid, S. V. Eck, Electron affinities of ${ }^{16,17,18} \mathrm{O}$, the fine structure of ${ }^{16} \mathrm{O}^{-}$and the hyperfine structure of ${ }^{17} \mathrm{O}^{-}$, Phys. Rev. A 64 (2001) 052504/1-14.

[14] T. Carette, C. Drag, O. Scharf, C. Blondel, C. Delsart, C. Froese Fischer, M. Godefroid, Isotope shift in the sulfur electron affinity: observation and theory, Phys. Rev. A 81 (2010) 042522.

[15] T. Carette, M. Godefroid, Theoretical study of the $\mathrm{C}^{-{ }^{4}} \mathrm{~S}_{3 / 2}$ and ${ }^{2} \mathrm{D}_{3 / 2,5 / 2}$ bound states and $\mathrm{C}$ ground configuration: Fine and hyperfine structures, isotope shifts, and transition probabilities, Phys. Rev. A 83 (2011) 062505 . 
[16] I. Grant, Relativistic atomic structure, in: G. Drake (Ed.), Atomic, Molecular and Optical Physics Handbook, Springer, Berlin, 2006, pp. $325-357$.

[17] R. D. Cowan, The Theory of Atomic Structure and Spectra, Los Alamos Series in Basic and Applied Sciences, University of California Press, 1981.

[18] P. Jönsson, C. Froese Fischer, SMS92: a program for relativistic isotope shift calculations, Comput. Phys. Commun. 100 (1997) 81-92.

[19] F. Parpia, C. Froese Fischer, I. Grant, Grasp92: A package for largescale relativistic atomic structure calculations, Comput. Phys. Commun. 94 (1996) 249-271.

[20] J. Li, C. Nazé, G. Gaigalas, P. Jönsson, M. Godefroid, On the breakdown of the Dirac kinetic energy operator for estimating the isotope normal mass shift, Eur. Phys. J. D (2012) in press.

[21] P. Mohr, B. Taylor, D. Newell, CODATA recommended values of the fundamental physical constants: 2006, Rev. Mod. Physics 80 (2008) 633.

[22] G. Torbohm, B. Fricke, A. Rosén, State-dependent volume isotope shifts of low-lying states of group-IIa and II-b elements, Phys. Rev. A 31 (1985) 2038-2053.

[23] S. Blundell, P. Baird, C. Palmer, D. Stacey, G. Woodgate, A reformulation of the theory of field isotope shift in atoms, J. Phys. B : At. Mol. Phys. 20 (1987) 3663-3681.

[24] E. C. Seltzer, K X-Ray Isotope Shifts, Phys. Rev. 188 (1969) 1916-1919.

[25] A. Borgoo, O. Scharf, G. Gaigalas, M. Godefroid, Multiconfiguration electron density function for the ATSP2K-package, Comput. Phys. Commun. 181 (2010) 426-439.

[26] G. Gaigalas, S. Fritzsche, I. Grant, Program to calculate pure angular momentum coefficients in $j j$-coupling, Comput. Phys. Commun. 139 (2001) 263-278. 
[27] G. Gaigalas, Z. Rudzikas, C. Froese Fischer, An efficient approach for spin-angular integrations in atomic structure calculations, J. Phys. B : At. Mol. Phys. 30 (1997) 3747-3771.

[28] I. Grant, Relativistic Quantum Theory of Atoms and Molecules. Theory and Computation, Atomic, Optical and Plasma Physics, Springer, New York, USA, 2007.

[29] E. Wigner, Group Theory and its Applications to the Quantum Mechanics of Atomic Spectra, Academic Press, New York, 1959.

[30] I. Angeli, A consistent set of nuclear rms charge radii: properties of the radius surface R(N,Z), At. Data Nucl. Data Tables 87 (2004) 185-206.

\section{Test run output}

Once installation is over, we recommend the user to run the test cases proposed in the subdirectory Grasp2K_v1_1/manual/test_ris. Therein, the directory "Input" contains two script files (scriptNd_2S and scriptNd_2P) that produce results files. The latter should match perfectly the ones already present in the "Output" directory.

The test run proposes to generate electron densities $(23)$, normal $(13)$ and specific mass shift (14) parameters for two states of neodymium $(Z=60)$ lithium-like, the ground state $1 s^{2} 2 s^{2} S_{1 / 2}$ (with scriptNd_2S) and the two excited states $1 s^{2} 2 s^{2} P_{1 / 2,3 / 2}$ (with scriptNd_2P). The purpose of these two cases is to show to the user how calculations are handled for a single level but also a case where several levels are optimized at the same time. A "readme_RIS" file is at the disposal of the interested user that desires details of the above calculations.

The contents of the file isodata are displayed in Fig. 1; those of clist . out appear in Fig. 2. In the following example, the default nuclear root mean square of the GRASP2K has not been used, instead one uses $\left\langle r^{2}\right\rangle^{1 / 2}=4.9118 \mathrm{fm}$ coming from Angeli's tables [30]. The session log for the test run has been captured in Fig. 3. A truncated form of the RIS3 Summary File for the test run appears in Fig. 4. Finally, the outputs of the programs RIS3_LSJ and RISOSUPPORT are displayed in Fig. 5 and Fig. 6, respectively. 


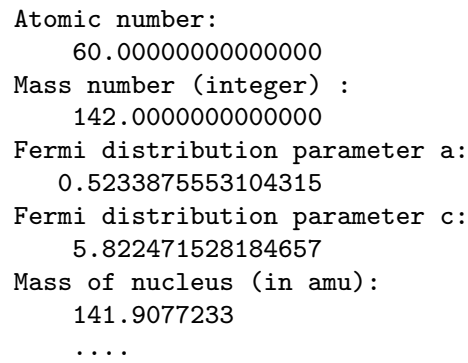

Figure 1: The GRASP2K isotope file isodata.

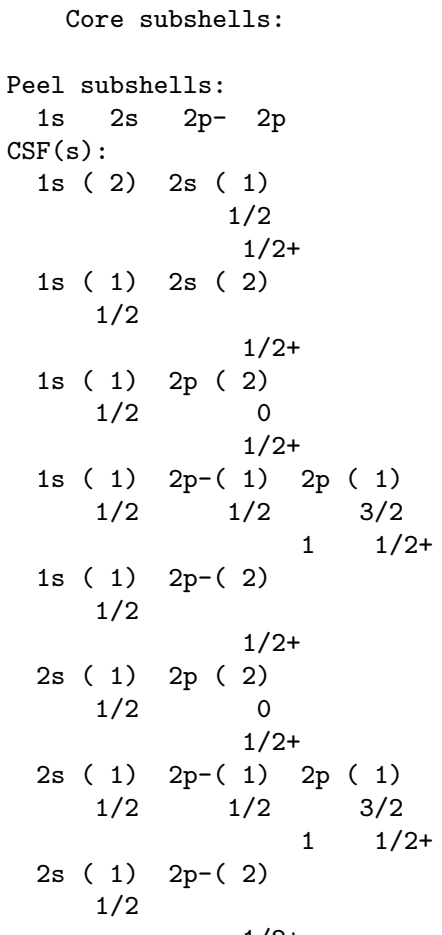

Figure 2: The GRASP2K Configuration Symmetry List File rcsl.out. 
\$ ris3

RIS: Execution begins ...

Default settings?

$\mathrm{y}$

Name of state

Nd1s2_n2

Mixing coefficients from a CI calc.?

Loading Configuration Symmetry List File ...

There are 4 relativistic subshells;

There are 8 relativistic CSFs;

... load complete;

Nd1s2_n2. wsetrwfa

Loading Radial WaveFunction File ...

$\begin{array}{lll}\text { nelec } & = & 3 \\ \text { ncftot } & = & 8 \\ \text { nw } & = & 4 \\ \text { nblock } & = & 1\end{array}$

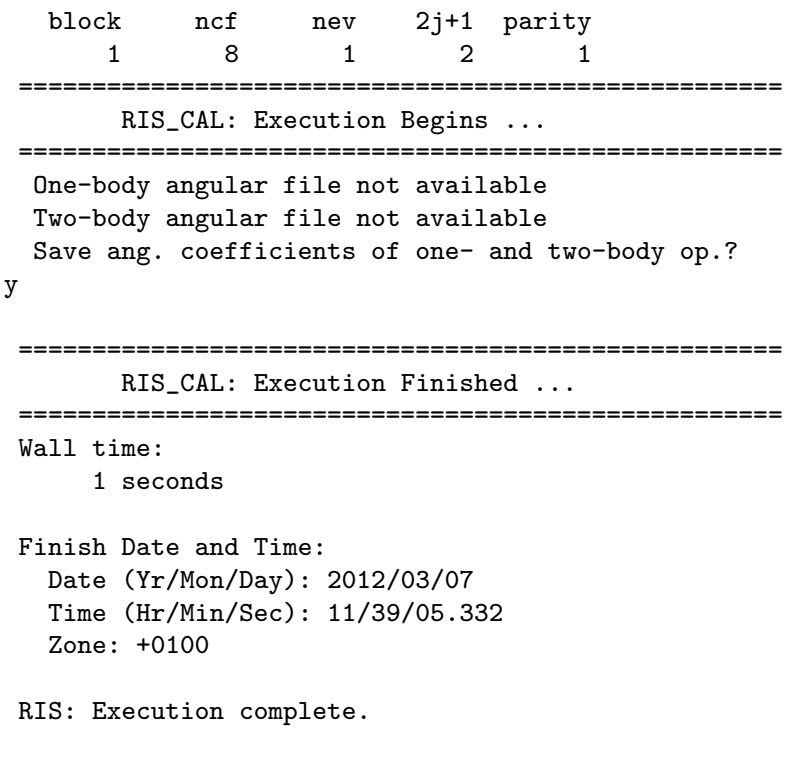

Figure 3: The RIS3 test run session log 


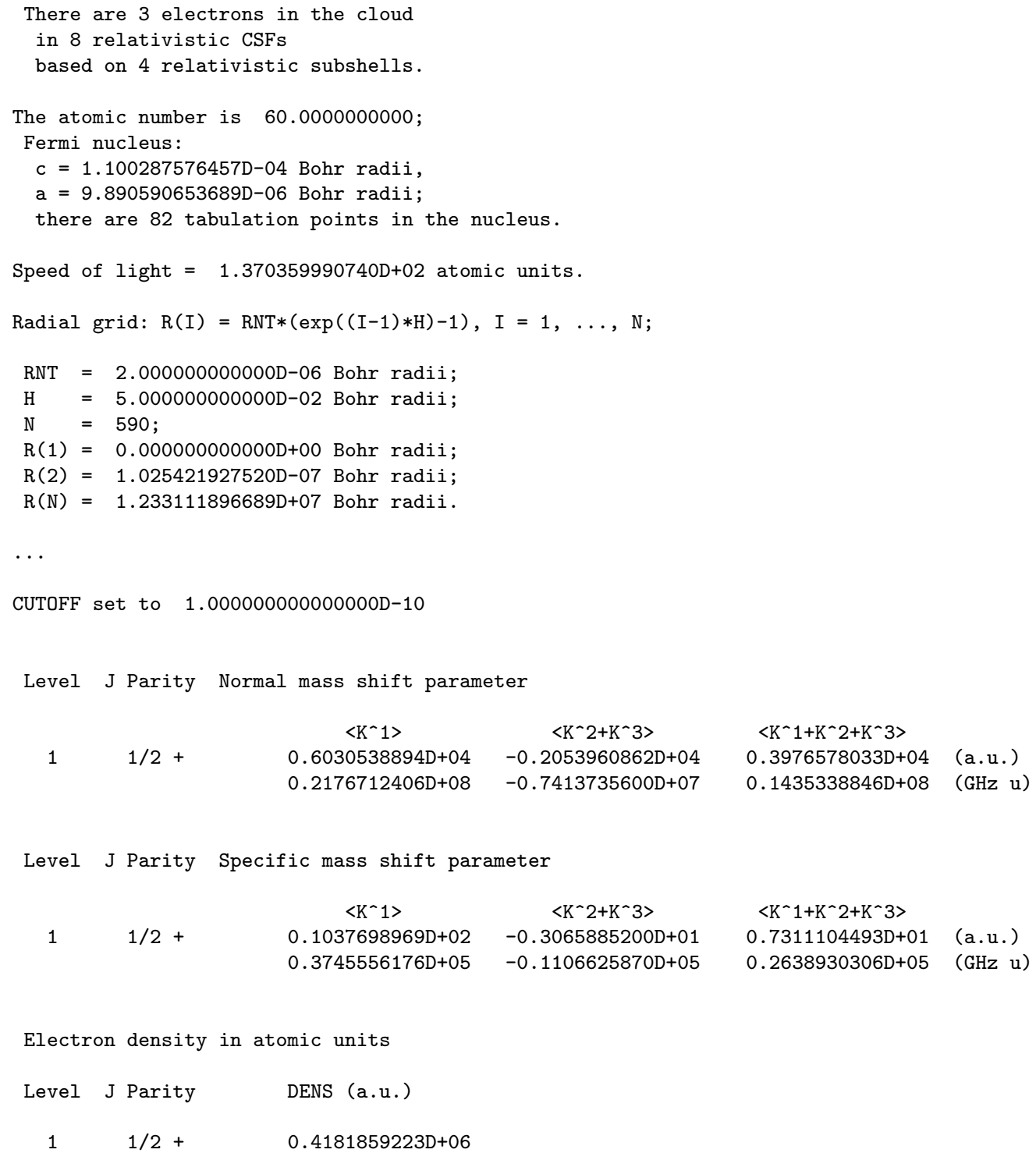

Figure 4: Test run output Nd2S_n2.i

$\begin{array}{rrrcrcc}\text { Energy } & \text { State } & \text { J } & \text { P } & \text { K_NMS (a.u.) } & \text { K_SMS (a.u.) } & \text { Dens (a.u.) } \\ -4204.7380729 & \text { 1s(2).2s_2S } & 1 / 2 & + & 3.9766011 D+03 & 7.7414916 D+00 & 4.1818792 D+05 \\ -4199.5777646 & \text { 1s(2).2p_2P } & 1 / 2 & - & 3.9729127 D+03 & -2.1929177 D+02 & 3.9134710 D+05 \\ -4177.5643106 & \text { 1s(2).2p_2P } & 3 / 2 & - & 3.9527821 D+03 & -2.3522968 D+02 & 3.9040259 D+05\end{array}$

Figure 5: Test run output of RIS3_LSJ 


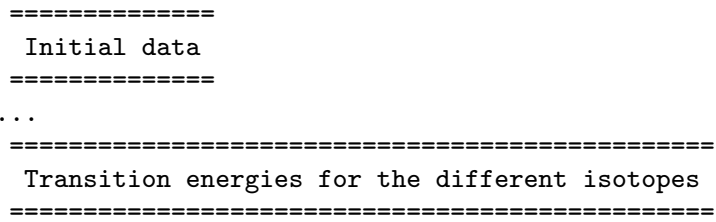

For 140Nd:

E_h
$5.1603228508 \mathrm{E}+00$

$\mathrm{eV}$

140.419536 $\mathrm{cm}-1$

$1.1325599256 \mathrm{E}+06$
$\mathrm{MHz}$

3. 3953292565E+10
Angstrom $8.8295550121 \mathrm{E}+01$

For 150Nd:
E_h
$5.1588011515 \mathrm{E}+00$

eV

$\mathrm{cm}-1$ $1.1322259512 \mathrm{E}+06$
$\mathrm{MHz}$ 3. $3943280264 \mathrm{E}+10$
Angstrom 8. 8321587259E+01

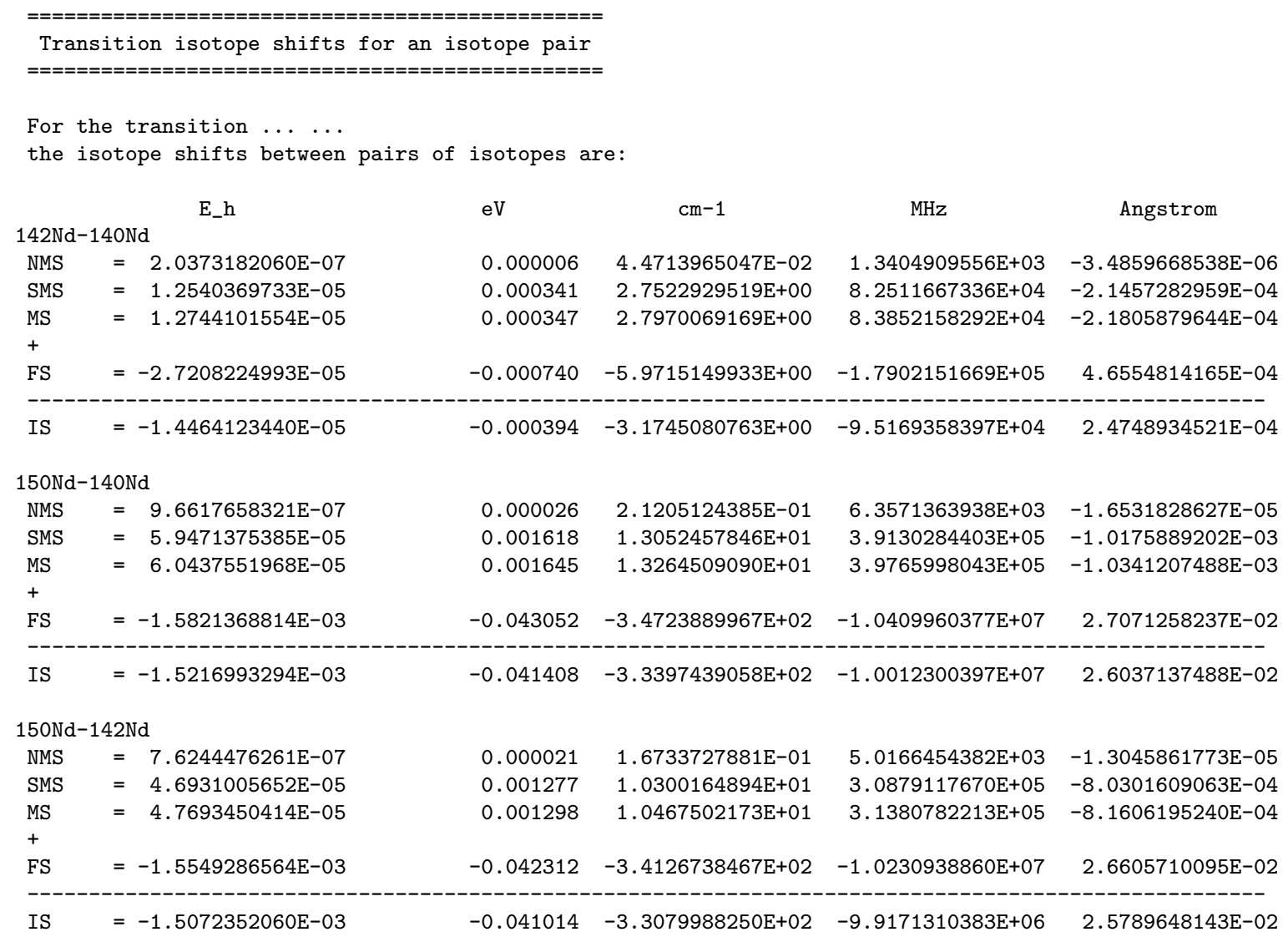

Figure 6: Test run output of RISOSUPPORT 